\title{
Produção de milho adubado residualmente com composto de lodo de esgoto e fosfato de Gafsa
}

\author{
G eraldo R. Zuba Junio ${ }^{1}$, Regynaldo A. Sampaio ${ }^{1}$, Altina L. N ascimento ${ }^{2}$, \\ João P. Carneiro ${ }^{1}$, Leonardo D. T. Santos ${ }^{1} \&$ Luiz A. Fernandes $^{1}$
}

\begin{abstract}
RESU MO
O bjetivou-se, com este trabalho, avaliar o efeito residual da adubação com composto de lodo de esgoto e fosfato natural de Gafsa sobre os teores de nutrientes no solo, na planta e na produtividade do milho. 0 trabalho foi realizado em Cambissolo $\mathrm{H}$ áplico. O s tratamentos, em esquema fatorial $2 \times 4$, corresponderam a 2 doses de fosfato de $\mathrm{G}$ afsa $\left(0\right.$ e $90 \mathrm{~kg} \mathrm{ha}^{-1}$ de $\mathrm{P}_{2} \mathrm{O}_{5}$ ) e 4 doses de composto de lodo de esgoto $\left(0 ; 25 ; 50\right.$ e $75 \mathrm{t} \mathrm{ha}^{-1}$, em base seca). 0 delineamento experimental utilizado foi em blocos casualizados, com 3 repetições. Em geral, a produtividade e os teores de nutrientes no solo e na planta no segundo cultivo sucessivo de milho não foram influenciados pela adubação com fosfato natural reativo. Entretanto, a produtividade de milho e os teores de nutrientes no solo e nas folhas de milho aumentaram com a dose de composto de lodo de esgoto ao solo, sendo a dose de $75 \mathrm{Mg} \mathrm{ha}^{-1}$, a de maior efeito residual. A produtividade do milho é menor no segundo cultivo sucessivo em razão do empobrecimento do solo em fósforo e potássio, recomendando-se novas adubações com lodo de esgoto, a cada cultivo realizado.
\end{abstract}

Palavras-chave: Zea mays, biossólido, fosfato natural reativo

\section{Corn yield response to residual effect of fertilization with sewage sludge compost and rock phosphate}

\begin{abstract}
The aim of this study was to evaluate the residual effect of fertilization with sewage sludge compost and rock phosphate on the chemical properties of the soil, nutrient content in plant and corn yield. The study was conducted on $\mathrm{H}$ aplic Cambisol. The treatments, in a factorial $2 \times 4$, corresponded to two doses of Gafsa phosphate ( 0 and $90 \mathrm{~kg} \mathrm{P}_{2} \mathrm{O}_{5}$ ha-1 $^{-1}$ ) and four doses of sewage sludge compost $\left(0,25,50\right.$ and $75 \mathrm{Mg} \mathrm{ha}^{-1}$ dry weight basis). The experimental design was in randomized blocks with three replications. In general, the corn yield and content of macronutrients in soil and plant on the second crop of corn were not influenced by the residual effects of fertilization with phosphate rock. How ever, corn yield and nutrient content in soil and leaves increased with the dose of sewage sludge compost applied to the soil, and the dose of $75 \mathrm{M} \mathrm{g} \mathrm{ha}^{-1}$ was the one with the highest residual effect. The yield of corn was lower in the second successive cultivation, due to the impoverishment of the soil phosphorus and potassium, recommending new fertilization with sewage sludge in every cultivation.
\end{abstract}

Key words: Zea mays, biosolids, reactive phosphate rock 


\section{INTRODUÇão}

A crescente implantação de estações de tratamento de esgotos nos municípios brasileiros tem ampliado enormemente o volume de lodo gerado, causando grande preocupação quanto à sua disposição final (Biondi \& Nascimento, 2005; Lemainski \& Silva, 2006b).

Apesar das várias alternativas existentes para o descarte final do lodo de esgoto, sua utilização na agricultura apresentase como a mais ecologicamente correta uma vez que promove a reciclagem de nutrientes e a melhoria física e química no solo, além de ser uma solução de longo alcance para destinação final deste resíduo, podendo ser utilizado no cultivo de grandes culturas, silvicultura, floricultura, paisagismo ou na recuperação de áreas degradadas (Barbosa et al., 2007). É uma importante fonte de matéria orgânica e de elementos essenciais às plantas podendo complementar os fertilizantes minerais e reduzir os custos de produção (Silva et al., 2002b; Biondi \& Nascimento, 2005; Lemainski \& Silva, 2006a).

No Brasil, o lodo de esgoto tem apresentado bons resultados em experimentos com cana-de-açúcar (Chiba et al., 2008a,b), feijão (Nascimento et al., 2004; Nogueira et al., 2006), milho (Nascimento et al., 2004; Trannin et al., 2005; Lemainski \& Silva, 2006a; Nogueira et al., 2006) e Soja (Vieira et al., 2005; Lemainski \& Silva, 2006b).

Nascimento et al. (2004) observaram aumento significativo na produtividade de milho e de feijão com o incremento das doses de lodo de esgoto associado, possivelmente, à melhoria das características químicas do solo. Por outro lado, Nogueira et al. (2006) observaram que as produções de milho e de feijão consorciados não diferiram quando a adubação foi feita com lodo de esgoto ou com fertilizante mineral. Segundo Silva et al. (2002a), o biossólido, por ser geralmente rico em nutrientes, pode ser utilizado para substituir, parcial ou totalmente, os adubos químicos.

O efeito residual da aplicação lodo de esgoto no solo tem influenciado positivamente a produtividade de milho (Barbosa et al., 2007; Silva et al., 2002b). Lemainski \& Silva (2006b) constataram que o efeito residual da aplicação de biossólido em cultivo de milho foi mais significativo na dose de $30 \mathrm{t} \mathrm{ha}^{-1}$, não diferindo da dose de $45 \mathrm{t} \mathrm{ha}^{-1}$, o que proporcionou uma produtividade de 77 e $80 \%$ das obtidas respectivamente no primeiro cultivo. As doses de biossólido também foram $22 \mathrm{e}$ $19 \%$ mais eficientes que o fertilizante mineral ressaltando, assim, a viabilidade da utilização do biossólido como fonte fornecedora de nutrientes às plantas, por prazos mais longos, corroborando com os resultados obtidos por Lemainski \& Silva (2006b) ao constatarem a viabilidade da aplicação de doses superiores a $30 \mathrm{t} \mathrm{ha}^{-1}$ de biossólido em soja os quais foram, em média, 18\% mais eficientes que o fertilizante mineral.

Objetivou-se, com o presente trabalho, avaliar o efeito residual da adubação com composto de lodo de esgoto e fosfato natural de Gafsa sobre os teores de nutrientes no solo, na planta e na produtividade do milho.

\section{MATERIAL E MÉTODOS}

O experimento foi realizado na área experimental do Campus da UFMG, em Montes Claros, MG, latitude $16^{\circ} 51^{\prime} 38^{\prime \prime} \mathrm{S}$ e longitude $44^{\circ} 55^{\prime} 00^{\prime \prime} \mathrm{W}$, em área de Cambissolo Háplico, com as seguintes características químicas e físicas da camada de 0$20 \mathrm{~cm}$, conforme metodologias preconizadas pela EMBRAPA (1997): Matéria orgânica $=1,09 \mathrm{dag} \mathrm{kg}^{-1} ; \mathrm{pH}$ em água $=5,5 ; \mathrm{P}-$ Mehlich 1=3,2 $\mathrm{mg} \mathrm{dm}^{-3}$; P-remanescente $=37,5 \mathrm{mg} \mathrm{L}^{-1} ; \mathrm{K}=67$ $\mathrm{mg} \mathrm{dm}{ }^{-3} ; \mathrm{Ca}=3,10 \mathrm{cmol}_{\mathrm{c}} \mathrm{dm}^{-3} ; \mathrm{Mg}=1,10 \mathrm{cmol}_{\mathrm{c}} \mathrm{dm}^{-3} ; \mathrm{Al}=0,50$ $\mathrm{cmol}_{\mathrm{c}} \mathrm{dm}^{-3} ; \mathrm{H}+\mathrm{Al}=4,94 \mathrm{cmol}_{\mathrm{c}} \mathrm{dm}^{-3} ;$ Soma de bases $=4,37$ $\mathrm{cmol}_{\mathrm{c}} \mathrm{dm}^{-3}$; CTC efetiva $=4,87 \mathrm{cmol}_{\mathrm{c}} \mathrm{dm}^{-3} ; \mathrm{m}=10 \%$; CTC total $=9,31 \mathrm{cmol} \mathrm{dm}^{-3} ; \mathrm{V}=47 \%$; Areia grossa $=13,30 \mathrm{dag} \mathrm{kg}^{-1}$; Areia fina $=24,70{ }^{\mathrm{c}} \mathrm{dag} \mathrm{kg} \mathrm{kg}^{-1} ;$ Silte $=30 \mathrm{dag} \mathrm{kg}^{-1}$ e Argila $=32 \mathrm{dag} \mathrm{kg}^{-1} . \mathrm{O}$ milho (Zea mays) variedade BR 106 foi cultivado em segundo cultivo sucessivo, no período de 10 de abril a 15 de setembro de 2010.

Os tratamentos, em esquema fatorial $2 \times 4$, corresponderam a 2 doses de fosfato natural reativo $\left(0\right.$ e $90 \mathrm{~kg} \mathrm{ha}^{-1}$ de $\left._{\mathrm{P}_{2}} \mathrm{O}_{5}\right)$ combinadas com 4 doses de composto de lodo de esgoto ( 0 ; 25; 50 e $75 \mathrm{t} \mathrm{ha}^{-1}$, em base seca), com três repetições, no delineamento em blocos casualizados.

O fosfato natural reativo utilizado foi o fosfato de Gafsa com as seguintes características químicas, conforme metodologias preconizadas por Tedesco et al. (1995): $\mathrm{P}_{2} \mathrm{O}_{5}$ total $=29 \% ; \mathrm{P}_{2} \mathrm{O}_{5}$ solúvel em ácido cítrico a $2 \%$ relação $1: 100=10 \%$; $\mathrm{P}_{2} \mathrm{O}_{5}$ solúvel em ácido fórmico a $2 \%$ relação $1: 100=21 \% ; \mathrm{SO}_{3}=$ $3,2 \% ; \mathrm{SiO}_{2}=3,6 \% ; \mathrm{Ca}=32 \% ; \mathrm{MgO}=0,8 \% ; \mathrm{K}_{2} \mathrm{O}=0,11 \%$. Calculou-se a dose aplicada com base no teor disponível de fósforo no solo e na recomendação de Noce (2004) para a variedade de milho BR 106.

O lodo de esgoto desidratado foi coletado na Estação de Tratamento de Esgoto - ETE no município de Juramento, MG. A ETE é operada pela COPASA-MG e possui capacidade para tratar $217 \mathrm{~m}^{3}$ por dia de esgoto. A linha de tratamento é composta por tratamento preliminar e reator anaeróbio UASB interligado em série a uma lagoa de pós-tratamento do tipo facultativa. $\mathrm{O}$ lodo gerado no reator UASB é desidratado em um leito de secagem e, posteriormente, disposto em um aterro controlado, implantado na área da estação. O lodo gerado possuía as seguintes características químicas, conforme metodologias preconizadas por Tedesco et al. (1995): $\mathrm{N}=1,77 ; \mathrm{P}=0,41 ; \mathrm{K}=$ 0,$66 ; \mathrm{Ca}=0,05 ; \mathrm{Mg}=0,22$ e $\mathrm{S}=1,24 \mathrm{dag} \mathrm{kg}^{-1}$.

Para a formulação do fertilizante orgânico a ser aplicado nas plantas de milho procedeu-se a uma compostagem realizada com o lodo de esgoto e palha de feijão, em que a última apresentava as seguintes características químicas, conforme metodologias preconizadas por Tedesco et al. (1995): $\mathrm{N}=0,91$; $\mathrm{P}=0,12 ; \mathrm{K}=2,00 ; \mathrm{Ca}=1,2 ; \mathrm{Mg}=0,40$ e $\mathrm{S}=0,04$ dag $\mathrm{kg}^{-1}$.

A mistura foi feita de forma a se obter uma relação $\mathrm{C} / \mathrm{N}$ de 30/1. A compostagem foi desenvolvida por meio de pilhas com altura de aproximadamente 1,5 m. Diariamente foram monitoradas a temperatura e a umidade. Para homogeneização do composto, controle de temperatura e aeração, efetuou-se o revolvimento manual das pilhas, utilizando-se pás e enxadas. O término do processo de compostagem foi identificado pelo final da etapa de maturação e obtenção de um material orgânico homogêneo em adiantada fase de humificação.

As doses de composto de lodo de esgoto foram baseadas na concentração de $\mathrm{N}$ neste adubo e na recomendação de adubação nitrogenada (Noce, 2004) para a variedade de milho BR 106 (80 kg ha-1 de N). O composto de lodo de esgoto 
apresentou as seguintes características químicas, conforme metodologias preconizadas por Tedesco et al. (1995): $\mathrm{N}=1,63$; $\mathrm{P}=0,42 ; \mathrm{K}=0,68 ; \mathrm{Ca}=0,40 ; \mathrm{Mg}=0,22 \mathrm{e} \mathrm{S}=1,26 \mathrm{dag} \mathrm{kg}^{-1}$.

A adubação foi realizada de uma única vez, em sulcos de plantio utilizando-se apenas o fosfato de Gafsa e o composto de lodo de esgoto, conforme os tratamentos. O espaçamento entre fileiras para a cultura de milho foi de $80 \mathrm{~cm}$ e semeio de 5 sementes por metro linear. $\mathrm{O}$ tamanho da parcela foi de $6,0 \mathrm{x}$ 4,8 m. Foram colhidas as quatro fileiras centrais de $4 \mathrm{~m}$ de comprimento, tendo-se eliminado as duas fileiras periféricas e $1 \mathrm{~m}$ de final da fileira como bordadura. O segundo plantio de milho foi feito 30 dias depois da colheita do primeiro, após uma capina manual e deposição do restolho sobre a parcela respectiva e sulcamento da área com enxadas. Os sulcos foram feitos sobre o local das linhas de plantio do primeiro cultivo para distribuição das sementes. Ao longo do ciclo a cultura sempre foi mantida no limpo. O método de irrigação utilizado foi o de aspersão, com turno de irrigação de 3 vezes por semana, determinando-se as lâminas com base nos dados de evaporação de água do tanque Classe A da Estação Climatológica do INMET, localizado a $500 \mathrm{~m}$ do local do experimento e nos coeficientes de cultura (Kc), conforme descritos em Albuquerque et al. (2000) para o cultivo do milho no norte de Minas.

No início do florescimento da cultura foram coletadas amostras de folhas em 20 plantas de cada unidade experimental, retirando-se a folha imediatamente abaixo e oposta à espiga, para análise dos teores de N, P, K, Ca, Mg e S (Tedesco et al., 1995; Malavolta et al., 1997).

Após a colheita avaliou-se a produtividade de grãos e se coletaram, entre plantas, nas profundidades de $0-10 ; 10-20 ; 20$ $30 ; 30-40$ e $40-50 \mathrm{~cm}, 30$ subamostras por parcela para formarem uma amostra composta, por profundidade, para análises de $\mathrm{P}$, K, Ca, Mg e S (EMBRAPA, 1997) e N mineral total (Tedesco et al., 1995).

Os dados obtidos foram submetidos à análise de variância, sendo as médias referentes às doses de fosfato testadas pelo teste de Tukey até $5 \%$ de probabilidade e as relativas às doses de composto de lodo de esgoto ajustadas aos modelos de regressão, testando-se os coeficientes até $10 \%$ de probabilidade pelo teste $\mathrm{t}$.

\section{RESULTADOS E DISCUSSÃO}

A análise de variância do segundo cultivo revelou que não houve interação entre as doses de fosfato e as de composto de lodo de esgoto, aplicadas antes do primeiro plantio de milho $(\mathrm{P}$ $>0,05)$.

Como se observa na Tabela 1 , não ocorreu efeito residual do fosfato natural de Gafsa sobre a produtividade do milho embora o teor inicial de $\mathrm{P}$ no solo fosse baixo, da ordem de 3,2 $\mathrm{mg} \mathrm{dm}^{-3}$. Pode-se atribuir tal fato à baixa taxa de solubilização do fosfato natural, em razão do teor inicial de cálcio no solo ser elevado, da ordem de $3,10 \mathrm{cmol}_{\mathrm{c}} \mathrm{dm}^{-3}$ e da água de irrigação apresentar pH em torno de 7,6. Resultado semelhante observaram Corrêa et al. (2005) em milho, cujo teor de cálcio mais elevado no solo afetou a solubilização do fosfato de Gafsa. Novais \& Smith (1999) também destacam que o baixo teor de cálcio no solo é fator preponderante para uma solubilização melhor de fosfatos naturais, uma vez que a baixa atividade de cálcio na solução do solo induz à maior liberação deste elemento do adubo. Entretanto, Resende et al. (2006) observaram que a produtividade de milho adubado com fosfato natural reativo foi superior no segundo cultivo quando comparado com o primeiro.

Apesar do tempo de contato do fosfato natural com o solo ser considerado fator importante na dissolução do mesmo, não foi constatado efeito residual um ano após a aplicação de 90 $\mathrm{kg} \mathrm{ha}^{-1}$ de $\mathrm{P}_{2} \mathrm{O}_{5}$, na forma de fosfato natural de Gafsa, sobre os teores de fósforo no solo (Tabela 1). De acordo com Novais et al. (2007) para os solos brasileiros, mesmo para aqueles com menor fator capacidade de fósforo, o efeito do tempo sobre o fosfato natural favorece a planta (P-disponível) e não o solo (fixação de $\mathrm{P}$ ) havendo, porém, redução da eficiência do fosfato natural comparado com as fontes fosfatadas mais solúveis.

Tal como ocorreu para o fósforo, os teores de N, K, Ca e Mg não foram influenciados pela aplicação de fosfato natural, exceto cálcio e magnésio, nas profundidades de 40-50 e 30-50 $\mathrm{cm}$, respectivamente (Tabelas 1 e 2). Esses resultados podem ser atribuídos à constituição do fosfato natural de Gafsa, que apresenta cálcio e magnésio em sua composição. Apesar da baixa taxa de solubilização pode ter havido alguma liberação e movimentação de cálcio e magnésio para as camadas mais profundas do solo.

Na Tabela 3 observa-se que no segundo cultivo do milho os teores de macronutrientes no tecido foliar não foram influenciados pela adubação fosfatada, resultados esperados uma vez que, em geral, o adubo fosfatado não influenciou os teores desses nutrientes no solo.

Observa-se, na Tabela 4, efeito residual do composto de lodo de esgoto um ano após sua aplicação no solo, em relação à produtividade de milho, com resposta linear para esta variável, a qual atingiu valor máximo dentro do intervalo experimental de 3,21 $\mathrm{Mg} \mathrm{ha}^{-1}$ de grãos, quando aplicados $75 \mathrm{Mg} \mathrm{ha}^{-1}$ de composto de lodo de esgoto. $\mathrm{O}$ efeito residual da aplicação de lodo de esgoto ao solo sobre a cultura do milho foi relatado também por Barbosa et al. (2007), Martins et al. (2003), Silva et al. (2002a) e Lemainski \& Silva (2006a). Esses últimos verificaram que doses de 30 e $45 \mathrm{Mg} \mathrm{ha}^{-1}$ de biossólido úmido apresentaram os melhores resultados proporcionando produtividades respectivas de 77 e $80 \%$ das obtidas no primeiro cultivo. Os autores constataram, ainda, que as doses de biossólido foram, em média, $21 \%$ mais eficientes que a do fertilizante mineral ressaltando, desta forma, a viabilidade da utilização do biossólido como fonte fornecedora de nutrientes às plantas.

Os teores de nitrogênio mineral em solo adubado com composto de lodo de esgoto e cultivado dois anos consecutivos com milho, decresceram com o incremento das doses de composto de lodo de esgoto (Tabela 4). Considerando que o aumento da dose de composto de lodo de esgoto resultou em aumentos lineares na produtividade da cultura, a redução deste nutriente no solo pode ser atribuída à exportação de nitrogênio pelos grãos que, de acordo com Duete et al. (2009), é superior a $100 \mathrm{~kg} \mathrm{ha}^{-1}$. Vieira et al. (2005) não constataram diferenças significativas nos teores de nitrogênio mineral no segundo cultivo de soja em solo adubado com composto de 
Tabela 1. Teores de macronutrientes primários no solo em função da adubação residual com fosfato de $\mathrm{G}$ afsa e composto de lodo de esgoto

\begin{tabular}{|c|c|c|c|c|c|c|}
\hline \multirow{2}{*}{ Variável } & \multirow{2}{*}{$\begin{array}{c}\text { Dose de } \\
\mathrm{P}_{2} \mathrm{O}_{5}\left(\mathrm{~kg} \mathrm{ha}^{-1}\right)\end{array}$} & \multicolumn{4}{|c|}{ Dose de composto de lodo de esgoto (Mg ha-1) } & \multirow{2}{*}{ Média } \\
\hline & & 0 & 25 & 50 & 75 & \\
\hline Produtividade (Mg ha- $\left.{ }^{-1}\right)$ & $\begin{array}{c}0 \\
90 \\
\text { Média }\end{array}$ & $\begin{array}{l}2,18 \\
2,16 \\
2,17\end{array}$ & $\begin{array}{l}2,93 \\
2,63 \\
2,78\end{array}$ & $\begin{array}{l}2,76 \\
2,97 \\
2,87\end{array}$ & $\begin{array}{l}3,36 \\
2,97 \\
3,17\end{array}$ & $\begin{array}{c}2,81 \mathrm{~A} \\
2,68 \mathrm{~A} \\
-\end{array}$ \\
\hline $\mathrm{N}(0-10 \mathrm{~cm})\left(\mathrm{mg} \mathrm{dm}^{-3}\right)$ & $\begin{array}{c}0 \\
90 \\
\text { Média }\end{array}$ & $\begin{array}{l}166,59 \\
185,22 \\
175,91\end{array}$ & $\begin{array}{l}146,05 \\
144,98 \\
145,52\end{array}$ & $\begin{array}{l}163,50 \\
113,37 \\
138,44\end{array}$ & $\begin{array}{l}109,69 \\
147,56 \\
128,63\end{array}$ & $\begin{array}{c}146,46 \mathrm{~A} \\
147,78 \mathrm{~A} \\
-\end{array}$ \\
\hline $\mathrm{N}(10-20 \mathrm{~cm})\left(\mathrm{mg} \mathrm{dm}^{-3}\right)$ & $\begin{array}{c}0 \\
90 \\
\text { Média }\end{array}$ & $\begin{array}{l}147,19 \\
166,42 \\
156,81\end{array}$ & $\begin{array}{l}114,75 \\
188,38 \\
151,57\end{array}$ & $\begin{array}{c}135,49 \\
89,16 \\
112,33\end{array}$ & $\begin{array}{l}117,67 \\
132,47 \\
125,07\end{array}$ & $\begin{array}{c}128,78 \mathrm{~A} \\
144,11 \mathrm{~A} \\
-\end{array}$ \\
\hline $\mathrm{N}(20-30 \mathrm{~cm})\left(\mathrm{mg} \mathrm{dm}^{-3}\right)$ & $\begin{array}{c}0 \\
90 \\
\text { Média }\end{array}$ & $\begin{array}{l}132,50 \\
125,08 \\
128,79\end{array}$ & $\begin{array}{l}179,20 \\
137,64 \\
158,42\end{array}$ & $\begin{array}{l}113,76 \\
103,92 \\
108,84\end{array}$ & $\begin{array}{l}144,02 \\
131,77 \\
137,90\end{array}$ & $\begin{array}{c}142,37 \mathrm{~A} \\
124,60 \mathrm{~A} \\
-\end{array}$ \\
\hline $\mathrm{N}(30-40 \mathrm{~cm})\left(\mathrm{mg} \mathrm{dm}^{-3}\right)$ & $\begin{array}{c}0 \\
90 \\
\text { Média }\end{array}$ & $\begin{array}{l}111,66 \\
118,67 \\
115,17\end{array}$ & $\begin{array}{l}182,75 \\
146,58 \\
164,67\end{array}$ & $\begin{array}{l}101,75 \\
130,12 \\
115,94\end{array}$ & $\begin{array}{l}121,79 \\
133,54 \\
127,67\end{array}$ & $\begin{array}{c}129,49 \mathrm{~A} \\
132,23 \mathrm{~A} \\
-\end{array}$ \\
\hline $\mathrm{N}(40-50 \mathrm{~cm})\left(\mathrm{mg} \mathrm{dm}^{-3}\right)$ & $\begin{array}{c}0 \\
90 \\
\text { Média }\end{array}$ & $\begin{array}{l}151,05 \\
184,08 \\
167,57\end{array}$ & $\begin{array}{l}200,04 \\
225,40 \\
212,72\end{array}$ & $\begin{array}{l}132,33 \\
118,58 \\
125,46\end{array}$ & $\begin{array}{l}122,91 \\
173,88 \\
148,40\end{array}$ & $\begin{array}{c}151,58 \mathrm{~A} \\
175,49 \mathrm{~A} \\
-\end{array}$ \\
\hline$P(0-10 \mathrm{~cm})\left(\mathrm{mg} \mathrm{dm}^{-3}\right)$ & $\begin{array}{c}0 \\
90 \\
\text { Média }\end{array}$ & $\begin{array}{r}5,47 \\
10,27 \\
7,87\end{array}$ & $\begin{array}{l}6,93 \\
8,13 \\
7,53\end{array}$ & $\begin{array}{l}8,83 \\
9,40 \\
9,12\end{array}$ & $\begin{array}{r}8,87 \\
11,40 \\
10,14\end{array}$ & $\begin{array}{c}7,53 \mathrm{~A} \\
9,80 \mathrm{~A} \\
-\end{array}$ \\
\hline$P(10-20 \mathrm{~cm})\left(\mathrm{mg} \mathrm{dm}^{-3}\right)$ & $\begin{array}{c}0 \\
90 \\
\text { Média }\end{array}$ & $\begin{array}{l}1,87 \\
7,47 \\
4,67\end{array}$ & $\begin{array}{l}3,77 \\
3,03 \\
3,40\end{array}$ & $\begin{array}{l}3,30 \\
5,17 \\
4,24\end{array}$ & $\begin{array}{l}2,03 \\
2,83 \\
2,43\end{array}$ & $\begin{array}{c}2,74 \mathrm{~A} \\
4,63 \mathrm{~A} \\
-\end{array}$ \\
\hline$P(20-30 \mathrm{~cm})\left(\mathrm{mg} \mathrm{dm}^{-3}\right)$ & $\begin{array}{c}0 \\
90 \\
\text { Média }\end{array}$ & $\begin{array}{l}0,70 \\
2,43 \\
1,57\end{array}$ & $\begin{array}{l}1,03 \\
0,80 \\
0,92\end{array}$ & $\begin{array}{l}1,23 \\
1,23 \\
1,23\end{array}$ & $\begin{array}{l}0,70 \\
1,07 \\
0,89\end{array}$ & $\begin{array}{c}0,92 \mathrm{~A} \\
1,38 \mathrm{~A} \\
-\end{array}$ \\
\hline$P(30-40 \mathrm{~cm})\left(\mathrm{mg} \mathrm{dm}^{-3}\right)$ & $\begin{array}{c}0 \\
90 \\
\text { Média }\end{array}$ & $\begin{array}{l}0,40 \\
2,20 \\
1,30\end{array}$ & $\begin{array}{l}0,47 \\
0,63 \\
0,55\end{array}$ & $\begin{array}{l}0,70 \\
0,77 \\
0,74\end{array}$ & $\begin{array}{l}0,57 \\
0,33 \\
0,45\end{array}$ & $\begin{array}{c}0,54 \mathrm{~A} \\
0,98 \mathrm{~A} \\
-\end{array}$ \\
\hline$P(40-50 \mathrm{~cm})\left(\mathrm{mg} \mathrm{dm}^{-3}\right)$ & $\begin{array}{c}0 \\
90 \\
\text { Média }\end{array}$ & $\begin{array}{l}0,40 \\
1,23 \\
0,82\end{array}$ & $\begin{array}{l}0,50 \\
0,47 \\
0,49\end{array}$ & $\begin{array}{l}0,50 \\
0,63 \\
0,57\end{array}$ & $\begin{array}{l}0,47 \\
0,47 \\
0,47\end{array}$ & $\begin{array}{c}0,47 \mathrm{~A} \\
0,70 \mathrm{~A} \\
-\end{array}$ \\
\hline $\mathrm{K}(0-10 \mathrm{~cm})\left(\mathrm{mg} \mathrm{dm}^{-3}\right)$ & $\begin{array}{c}0 \\
90 \\
\text { Média }\end{array}$ & $\begin{array}{r}121,33 \\
96,33 \\
108,83\end{array}$ & $\begin{array}{r}113,33 \\
84,67 \\
99,00\end{array}$ & $\begin{array}{l}101,33 \\
110,67 \\
106,00\end{array}$ & $\begin{array}{l}93,67 \\
80,00 \\
86,84\end{array}$ & $\begin{array}{c}107,42 \mathrm{~A} \\
92,92 \mathrm{~A} \\
-\end{array}$ \\
\hline $\mathrm{K}(10-20 \mathrm{~cm})\left(\mathrm{mg} \mathrm{dm}^{-3}\right)$ & $\begin{array}{c}0 \\
90 \\
\text { Média }\end{array}$ & $\begin{array}{l}65,00 \\
48,67 \\
56,84\end{array}$ & $\begin{array}{l}55,67 \\
43,00 \\
49,34\end{array}$ & $\begin{array}{l}52,00 \\
74,67 \\
63,34\end{array}$ & $\begin{array}{l}41,67 \\
43,67 \\
42,67\end{array}$ & $\begin{array}{c}53,59 \mathrm{~A} \\
52,50 \mathrm{~A} \\
-\end{array}$ \\
\hline $\mathrm{K}(20-30 \mathrm{~cm})\left(\mathrm{mg} \mathrm{dm}^{-3}\right)$ & $\begin{array}{c}0 \\
90 \\
\text { Média }\end{array}$ & $\begin{array}{l}32,33 \\
27,00 \\
29,67\end{array}$ & $\begin{array}{l}36,00 \\
29,00 \\
32,50\end{array}$ & $\begin{array}{l}29,67 \\
48,67 \\
39,17\end{array}$ & $\begin{array}{l}22,33 \\
26,33 \\
24,33\end{array}$ & $\begin{array}{c}30,08 \mathrm{~A} \\
32,75 \mathrm{~A} \\
-\end{array}$ \\
\hline $\mathrm{K}(30-40 \mathrm{~cm})\left(\mathrm{mg} \mathrm{dm}^{-3}\right)$ & $\begin{array}{c}0 \\
90 \\
\text { Média }\end{array}$ & $\begin{array}{l}22,33 \\
22,00 \\
22,17\end{array}$ & $\begin{array}{l}21,33 \\
23,33 \\
22,33\end{array}$ & $\begin{array}{l}20,67 \\
41,00 \\
30,84\end{array}$ & $\begin{array}{l}19,00 \\
16,00 \\
17,50\end{array}$ & $\begin{array}{c}20,83 \mathrm{~A} \\
25,58 \mathrm{~A} \\
-\end{array}$ \\
\hline $\mathrm{K}(40-50 \mathrm{~cm})\left(\mathrm{mg} \mathrm{dm}^{-3}\right)$ & $\begin{array}{c}0 \\
90 \\
\text { Média }\end{array}$ & $\begin{array}{l}19,33 \\
22,00 \\
20,67\end{array}$ & $\begin{array}{l}18,00 \\
23,33 \\
20,67\end{array}$ & $\begin{array}{l}20,33 \\
41,00 \\
30,67\end{array}$ & $\begin{array}{l}19,00 \\
16,00 \\
17,50\end{array}$ & $\begin{array}{c}19,17 \mathrm{~A} \\
25,58 \mathrm{~A} \\
-\end{array}$ \\
\hline
\end{tabular}

Para cada variável médias seguidas de mesma letra na vertical não diferem estatisticamente entre si pelo teste Tukey a 0,05 de probabilidade

lodo de esgoto, possivelmente associados à extração e à exportação deste elemento pelos cultivos.

Os teores de fósforo no solo aumentaram com o aumento das doses de composto de lodo de esgoto, na camada de $0-10 \mathrm{~cm}$ (Tabela 4) mesmo que não se tenha verificado efeito da aplicação do composto de lodo de esgoto sobre os teores deste elemento, na camada de 10-30 $\mathrm{cm}$ nem constatado reduções em suas concentrações, na camada de $30-50 \mathrm{~cm}$. O efeito residual do lodo de esgoto no aumento dos teores de $\mathrm{P}$ no solo foi relatado por Barbosa et al. (2007; 2002). Silva et al. (2002a) também constataram que, três anos após a aplicação de lodo de esgoto e três cultivos consecutivos de milho, os teores de fósforo no solo eram maiores que os teores observados inicialmente.

De acordo com Novais et al. (2007) o P pode ser adsorvido 
Tabela 2. Teores de macronutrientes secundários no solo, em função da adubação residual com fosfato de Gafsa e composto de lodo de esgoto em milho

\begin{tabular}{|c|c|c|c|c|c|c|}
\hline \multirow{3}{*}{ Macronutrientes } & \multirow{3}{*}{$\begin{array}{l}\mathrm{P}_{2} \mathrm{O}_{5} \\
\left(\mathrm{~kg} \mathrm{ha}^{-1}\right)\end{array}$} & \multicolumn{4}{|c|}{$\begin{array}{l}\text { Dose de composto de } \\
\text { lodo de esgoto ( } \mathrm{Mg} \mathrm{ha}^{-1} \text { ) }\end{array}$} & \multirow{3}{*}{ Média } \\
\hline & & 0 & 25 & 50 & 75 & \\
\hline & & \multicolumn{4}{|c|}{$\mathrm{cmol}_{\mathrm{c}} \mathrm{dm}^{-3}$} & \\
\hline $\mathrm{Ca}(0-10 \mathrm{~cm})$ & $\begin{array}{c}0 \\
90 \\
\text { Média }\end{array}$ & $\begin{array}{l}5,47 \\
5,10 \\
5,29\end{array}$ & $\begin{array}{l}6,43 \\
5,70 \\
6,07\end{array}$ & $\begin{array}{l}6,23 \\
5,87 \\
6,05\end{array}$ & $\begin{array}{l}5,53 \\
5,46 \\
5,50\end{array}$ & $\begin{array}{l}5,92 \mathrm{~A} \\
5,53 \mathrm{~A}\end{array}$ \\
\hline Ca $(10-20 \mathrm{~cm})$ & $\begin{array}{c}0 \\
90 \\
\text { Média }\end{array}$ & $\begin{array}{l}3,00 \\
3,23 \\
3,12\end{array}$ & $\begin{array}{l}4,10 \\
3,03 \\
3,57\end{array}$ & $\begin{array}{l}4,10 \\
4,07 \\
4,09\end{array}$ & $\begin{array}{l}3,10 \\
2,97 \\
3,04\end{array}$ & $\begin{array}{c}3,58 \mathrm{~A} \\
3,33 \mathrm{~A} \\
-\end{array}$ \\
\hline $\mathrm{Ca}(20-30 \mathrm{~cm})$ & $\begin{array}{c}0 \\
90 \\
\text { Média }\end{array}$ & $\begin{array}{l}1,33 \\
1,83 \\
1,58\end{array}$ & $\begin{array}{l}2,10 \\
1,33 \\
1,72\end{array}$ & $\begin{array}{l}2,13 \\
1,93 \\
2,03\end{array}$ & $\begin{array}{l}1,57 \\
1,47 \\
1,52\end{array}$ & $\begin{array}{c}1,78 \mathrm{~A} \\
1,64 \mathrm{~A} \\
-\end{array}$ \\
\hline $\mathrm{Ca}(30-40 \mathrm{~cm})$ & $\begin{array}{c}0 \\
90 \\
\text { Média }\end{array}$ & $\begin{array}{l}0,77 \\
1,10 \\
0,94\end{array}$ & $\begin{array}{l}1,13 \\
1,26 \\
1,20\end{array}$ & $\begin{array}{l}1,36 \\
1,30 \\
1,33\end{array}$ & $\begin{array}{l}1,00 \\
0,97 \\
0,99\end{array}$ & $\begin{array}{l}1,07 \mathrm{~A} \\
1,16 \mathrm{~A}\end{array}$ \\
\hline $\mathrm{Ca}(40-50 \mathrm{~cm})$ & $\begin{array}{c}0 \\
90 \\
\text { Média }\end{array}$ & $\begin{array}{l}0,50 \\
0,80 \\
0,65\end{array}$ & $\begin{array}{l}0,80 \\
1,20 \\
1,00\end{array}$ & $\begin{array}{l}0,80 \\
1,50 \\
1,15\end{array}$ & $\begin{array}{l}0,60 \\
0,60 \\
0,60\end{array}$ & $\begin{array}{c}0,68 \mathrm{~B} \\
1,03 \mathrm{~A} \\
-\end{array}$ \\
\hline $\mathrm{Mg}(0-10 \mathrm{~cm})$ & $\begin{array}{c}0 \\
90 \\
\text { Média }\end{array}$ & $\begin{array}{l}1,07 \\
1,10 \\
1,09\end{array}$ & $\begin{array}{l}1,17 \\
1,10 \\
1,14\end{array}$ & $\begin{array}{l}1,20 \\
1,30 \\
1,25\end{array}$ & $\begin{array}{l}1,10 \\
1,17 \\
1,14\end{array}$ & $\begin{array}{c}1,14 \mathrm{~A} \\
1,17 \mathrm{~A} \\
-\end{array}$ \\
\hline $\mathrm{Mg}(10-20 \mathrm{~cm})$ & $\begin{array}{c}0 \\
90 \\
\text { Média }\end{array}$ & $\begin{array}{l}0,80 \\
0,87 \\
0,84\end{array}$ & $\begin{array}{l}0,93 \\
0,70 \\
0,82\end{array}$ & $\begin{array}{l}0,93 \\
0,93 \\
0,93\end{array}$ & $\begin{array}{l}0,67 \\
0,80 \\
0,74\end{array}$ & $\begin{array}{l}0,83 \mathrm{~A} \\
0,83 \mathrm{~A}\end{array}$ \\
\hline $\mathrm{Mg}(20-30 \mathrm{~cm})$ & $\begin{array}{c}0 \\
90 \\
\text { Média }\end{array}$ & $\begin{array}{l}0,40 \\
0,53 \\
0,47\end{array}$ & $\begin{array}{l}0,57 \\
0,43 \\
0,50\end{array}$ & $\begin{array}{l}0,57 \\
0,53 \\
0,55\end{array}$ & $\begin{array}{l}0,37 \\
0,47 \\
0,42\end{array}$ & $\begin{array}{l}0,48 \mathrm{~A} \\
0,49 \mathrm{~A}\end{array}$ \\
\hline $\mathrm{Mg}(30-40 \mathrm{~cm})$ & $\begin{array}{c}0 \\
90 \\
\text { Média }\end{array}$ & $\begin{array}{l}0,20 \\
0,40 \\
0,30\end{array}$ & $\begin{array}{l}0,30 \\
0,30 \\
0,30\end{array}$ & $\begin{array}{l}0,30 \\
0,40 \\
0,35\end{array}$ & $\begin{array}{l}0,20 \\
0,30 \\
0,25\end{array}$ & $\begin{array}{c}0,25 \mathrm{~B} \\
0,35 \mathrm{~A} \\
-\end{array}$ \\
\hline $\mathrm{Mg}(40-50 \mathrm{~cm})$ & $\begin{array}{c}0 \\
90 \\
\text { Média }\end{array}$ & $\begin{array}{l}0,20 \\
0,30 \\
0,25\end{array}$ & $\begin{array}{l}0,20 \\
0,30 \\
0,25\end{array}$ & $\begin{array}{l}0,27 \\
0,40 \\
0,34\end{array}$ & $\begin{array}{l}0,10 \\
0,23 \\
0,17\end{array}$ & $\begin{array}{c}0,19 \mathrm{~B} \\
0,31 \mathrm{~A} \\
-\end{array}$ \\
\hline
\end{tabular}

Médias seguidas da mesma letra na vertical não diferem estatisticamente entre si pelo teste Tukey a 0,05 de probabilidade

às partículas do solo resultando em baixa taxa de difusão do mesmo, o que explicaria o aumento dos teores deste elemento na camada superficial (Tabela 4). Levando-se em consideração o aumento da produtividade do milho e a profundidade efetiva do sistema radicular da cultura que, segundo Bordin et al. (2008), atinge profundidades superiores às avaliadas, com $80 \%$ do sistema radicular concentrando-se de 40 a $46 \mathrm{~cm}$, área em que ocorreu redução nos teores do elemento, o decréscimo em profundidade nos teores de $\mathrm{P}$ em parcelas que receberam maiores doses do composto poderia ser resultado da não movimentação do $\mathrm{P}$ adicionado, via composto de lodo de esgoto, no perfil do solo, e da maior absorção deste elemento em maiores profundidades por plantas de milho. Apesar do aumento linear de P na camada superficial do solo, a maior dose de composto aplicada foi insuficiente para alterar a classe de disponibilidade que, conforme Alvarez et al. (1999), tanto antes do primeiro cultivo quanto após o segundo cultivo do milho foi classificada como muito baixa.

Similar ao observado para o nitrogênio, os teores de potássio no solo, na camada de $0-10 \mathrm{~cm}$, diminuíram com o aumento da dose de composto de lodo de esgoto (Tabela 4). Nas camadas
Tabela 3. Teores de nutrientes na folha de milho em função da adubação residual com fosfato de Gafsa e composto de lodo de esgoto em milho

\begin{tabular}{|c|c|c|c|c|c|c|}
\hline \multirow{3}{*}{ Variável } & \multicolumn{5}{|c|}{$\begin{array}{l}\text { Dose de composto de lodo de esgoto } \\
\qquad\left(\mathrm{Mg} \mathrm{ha}^{-1}\right)\end{array}$} & \multirow{3}{*}{ Média } \\
\hline & \multirow[t]{2}{*}{$\left(\mathrm{kg} \mathrm{ha}^{-1}\right)$} & 0 & 25 & 50 & 75 & \\
\hline & & \multicolumn{4}{|c|}{ dag $\mathrm{kg}^{-1}$} & \\
\hline $\mathrm{N}$ & $\begin{array}{c}0 \\
90 \\
\text { Média }\end{array}$ & $\begin{array}{l}1,38 \\
1,22 \\
1,30\end{array}$ & $\begin{array}{l}1,48 \\
1,53 \\
1,51\end{array}$ & $\begin{array}{l}1,40 \\
1,47 \\
1,44\end{array}$ & $\begin{array}{l}1,66 \\
1,61 \\
1,64\end{array}$ & $\begin{array}{c}1,48 \mathrm{~A} \\
1,46 \mathrm{~A} \\
-\end{array}$ \\
\hline$P$ & $\begin{array}{c}0 \\
90 \\
\text { Média }\end{array}$ & $\begin{array}{l}0,20 \\
0,20 \\
0,20\end{array}$ & $\begin{array}{l}0,20 \\
0,30 \\
0,25\end{array}$ & $\begin{array}{l}0,20 \\
0,30 \\
0,25\end{array}$ & $\begin{array}{l}0,30 \\
0,30 \\
0,30\end{array}$ & $\begin{array}{c}0,23 \mathrm{~A} \\
0,28 \mathrm{~A} \\
-\end{array}$ \\
\hline K & $\begin{array}{c}0 \\
90 \\
\text { Média }\end{array}$ & $\begin{array}{l}0,83 \\
1,05 \\
0,94\end{array}$ & $\begin{array}{l}1,04 \\
1,13 \\
1,09\end{array}$ & $\begin{array}{l}1,03 \\
0,93 \\
0,98\end{array}$ & $\begin{array}{l}0,96 \\
0,91 \\
0,94\end{array}$ & $\begin{array}{c}0,97 \mathrm{~A} \\
1,01 \mathrm{~A} \\
-\end{array}$ \\
\hline $\mathrm{Ca}$ & $\begin{array}{c}0 \\
90 \\
\text { Média }\end{array}$ & $\begin{array}{l}0,52 \\
0,63 \\
0,58\end{array}$ & $\begin{array}{l}0,59 \\
0,60 \\
0,60\end{array}$ & $\begin{array}{l}0,65 \\
0,62 \\
0,64\end{array}$ & $\begin{array}{l}0,66 \\
0,66 \\
0,66\end{array}$ & $\begin{array}{c}0,61 \mathrm{~A} \\
0,63 \mathrm{~A} \\
-\end{array}$ \\
\hline $\mathrm{Mg}$ & $\begin{array}{c}0 \\
90 \\
\text { Média }\end{array}$ & $\begin{array}{l}0,09 \\
0,12 \\
0,11\end{array}$ & $\begin{array}{l}0,14 \\
0,16 \\
0,15\end{array}$ & $\begin{array}{l}0,12 \\
0,11 \\
0,12\end{array}$ & $\begin{array}{l}0,16 \\
0,14 \\
0,15\end{array}$ & $\begin{array}{c}0,13 \mathrm{~A} \\
0,13 \mathrm{~A} \\
-\end{array}$ \\
\hline$S$ & $\begin{array}{c}0 \\
90 \\
\text { Média }\end{array}$ & $\begin{array}{l}0,13 \\
0,12 \\
0,13\end{array}$ & $\begin{array}{l}0,13 \\
0,13 \\
0,13\end{array}$ & $\begin{array}{l}0,12 \\
0,12 \\
0,12\end{array}$ & $\begin{array}{l}0,14 \\
0,16 \\
0,15\end{array}$ & $\begin{array}{r}0,13 \mathrm{~A} \\
0,13 \mathrm{~A} \\
-\end{array}$ \\
\hline
\end{tabular}

Nota: Médias seguidas da mesma letra na vertical não diferem estatisticamente entre si pelo teste Tukey a 0,05 de probabilidade

de 20-30 e 30-40 cm, observou-se aumento deste elemento apenas até a dose máxima de $35 \mathrm{Mg} \mathrm{ha}^{-1}$. Ao fato do aumento das doses de composto de lodo de esgoto ter resultado em maior produtividade nos dois cultivos de milho e, consequentemente, maior exportação do nutriente, soma-se a rápida disponibilização do nutriente no solo de vez que este não integra nenhum composto orgânico estável (Ernani et al., 2007), não sendo esperado, portanto, efeito residual da aplicação de composto de lodo de esgoto para este nutriente.

Apesar da redução de potássio no solo no segundo cultivo de milho, com o aumento das doses de composto de lodo de esgoto, a disponibilidade deste elemento na camada superficial foi considerada boa enquanto seu teor inicial no solo antes da implantação dos tratamentos era médio. Corroborando com os resultados obtidos nesta pesquisa, Silva et al. (2001) constataram redução nos teores de potássio no solo com o aumento das doses de lodo de esgoto até os 484 dias de avaliação. Contudo, Barbosa et al. (2002) e Barbosa et al. (2007) constataram que o efeito residual da aplicação de doses crescentes de lodo de esgoto ao solo não influenciou os teores de potássio do solo, até a dose máxima aplicada de $36 \mathrm{Mg} \mathrm{ha}^{-1}$. Silva et al. (2002a) também observaram que, após 3 cultivos consecutivos de milho em área que recebeu uma única aplicação de doses de lodo de esgoto, o teor de potássio no solo permaneceu igual ao teor inicial.

Os teores de cálcio no solo aumentaram em todas as profundidades estudadas até a dose aproximada de $39 \mathrm{Mg} \mathrm{ha}^{-1}$ de composto de lodo de esgoto. Mesmo após o segundo cultivo de milho o teor deste elemento no solo, que foi de 5,49 $\mathrm{mg} \mathrm{dm}^{-3}$, com a aplicação da dose de máxima produtividade do milho, não retornou aos níveis de disponibilidade encontrados 
Tabela 4. Equações de regressão relacionando os teores de macronutrientes no solo e as doses residuais de composto de lodo de esgoto aplicadas

\begin{tabular}{|c|c|c|c|c|c|c|c|}
\hline Variável & Unidade & Equação & $\mathbf{R}^{2}$ & DC $\left(M_{g} h^{-1}\right)$ & TNS & TMP & $\mathrm{CL}^{1}$ \\
\hline Produtividade & $\mathrm{Mg} \mathrm{ha}^{-1}$ & $Y=2,28+0,01236 * X$ & 0,9040 & 75,00 & 3,21 & - & - \\
\hline $\mathrm{N}(0-10 \mathrm{~cm})$ & & $Y=169,52-0,59892 * X$ & 0,8906 & 0,00 & 169,52 & 124,60 & - \\
\hline$N(10-20 \mathrm{~cm})$ & & $Y=(24535,25-148,784198 * * * X)^{0,5}$ & 0,6447 & 0,00 & 156,64 & 115,66 & - \\
\hline$N(20-30 \mathrm{~cm})$ & $m g d m^{-1}$ & $Y=Y m=133,49$ & - & - & 133,49 & 133,49 & - \\
\hline $\mathrm{N}(30-40 \mathrm{~cm})$ & & $Y=Y m=130,86$ & - & - & 130,86 & 130,86 & - \\
\hline$N(40-50 \mathrm{~cm})$ & & $Y=Y m=163,54$ & - & - & 163,54 & 163,54 & - \\
\hline$P(0-10 \mathrm{~cm})$ & & $Y=\left(58,40+0,008116362 * * * X^{2}\right)^{0,5}$ & 0,9282 & 75,00 & 10,20 & 10,20 & MBx \\
\hline$P(10-20 \mathrm{~cm})$ & & $Y=Y m=3,69$ & - & - & 3,69 & 3,69 & MBX \\
\hline$P(20-30 \mathrm{~cm})$ & $\mathrm{mg} \mathrm{dm}^{-3}$ & $Y=Y m=1,15$ & - & - & 1,15 & 1,15 & MBX \\
\hline$P(30-40 \mathrm{~cm})$ & & $Y=1,23-0,0910886 * X^{0,5}$ & 0,8169 & 0,00 & 1,23 & 0,44 & MBX \\
\hline$P(40-50 \mathrm{~cm})$ & & $Y=\left(0,63-0,05045891^{\circ} X^{0,5}\right)^{05}$ & 0,7502 & 0,00 & 0,79 & 0,43 & MBX \\
\hline $\mathrm{K}(0-10 \mathrm{~cm})$ & & $Y=\left(11452,82-0,615897 * * * X^{2}\right)^{0,5}$ & 0,6888 & 0,00 & 107,02 & 89,38 & $B$ \\
\hline $\mathrm{K}(10-20 \mathrm{~cm})$ & & $Y=Y m=53,05$ & - & - & 53,05 & 53,05 & M \\
\hline$K(20-30 \mathrm{~cm})$ & $\mathrm{mg} \mathrm{dm}^{-3}$ & $Y=\left(794,19+32,00027 * * * X-0,447312 * * * X^{2}\right)^{0,5}$ & 0,7280 & 35,77 & 36,97 & 26,04 & $\mathrm{Bx}$ \\
\hline$K(30-40 \mathrm{~cm})$ & & $Y=20,66+0,383^{\circ} X-0,0054^{*} X^{2}$ & 0,5079 & 35,46 & 27,45 & 19,01 & Bx \\
\hline $\mathrm{K}(40-50 \mathrm{~cm})$ & & $Y=Y m=22,38$ & - & - & 22,38 & 22,38 & Bx \\
\hline $\mathrm{Ca}(0-10 \mathrm{~cm})$ & & $Y=5,30+0,04234 * X-0,000532 * X^{2}$ & 0,9922 & 39,79 & 6,15 & 5,49 & MB \\
\hline $\mathrm{Ca}(10-20 \mathrm{~cm})$ & & $Y=3,04+0,04612^{\circ} X-0,0006^{\circ} X^{2}$ & 0,8081 & 38,43 & 3,92 & 3,12 & B \\
\hline $\mathrm{Ca}(20-30 \mathrm{~cm})$ & $\mathrm{cmol}_{\mathrm{c}} \mathrm{dm}^{-3}$ & $Y=\left(2,31+0,070593 * * * X-0,000909 * * * X^{2}\right)^{0,5}$ & 0,6765 & 38,83 & 1,92 & 1,57 & M \\
\hline $\mathrm{Ca}(30-40 \mathrm{~cm})$ & & $Y=0,92+0,01912 * X-0,00024 * X^{2}$ & 0,9420 & 39,83 & 1,30 & 1,01 & $\mathrm{Bx}$ \\
\hline $\mathrm{Ca}(40-50 \mathrm{~cm})$ & & $Y=0,63+0,027 * X-0,00036 * X^{2}$ & 0,9419 & 37,50 & 1,13 & 0,63 & Bx \\
\hline $\mathrm{Mg}(0-10 \mathrm{~cm})$ & & $Y=1,08+0,00584 * X-0,000064 * X^{2}$ & 0,7139 & 45,63 & 1,21 & 1,15 & B \\
\hline$M g(10-20 \mathrm{~cm})$ & & $Y=Y m=0,83$ & - & - & 0,83 & 0,83 & M \\
\hline $\mathrm{Mg}(20-30 \mathrm{~cm})$ & $\mathrm{Cmol}_{\mathrm{c}} \mathrm{dm}^{-3}$ & $Y=Y m=0,49$ & - & - & 0,49 & 0,49 & M \\
\hline $\mathrm{Mg}(30-40 \mathrm{~cm})$ & & $Y=Y m=0,30$ & - & - & 0,30 & 0,30 & B \\
\hline $\mathrm{Mg}(40-50 \mathrm{~cm})$ & & $Y=Y m=0,25$ & - & - & 0,25 & 0,25 & B \\
\hline
\end{tabular}

DC - dose de composto de lodo de esgoto para atingir a maior produtividade ou a maior concentração do nutriente no solo, dentro do intervalo experimental

TNS - teor máximo do nutriente no solo, dentro do intervalo experimental

TMP - teor no solo com a aplicação da dose de composto de lodo de esgoto que gerou a máxima produtividade de milho

${ }^{1}$ Classes de fertilidade segundo Alvarez et al. (1999): A - alto, MB - muito bom, B - bom, M - médio, B - baixo, MBx - muito baixo

$\circ, * *, * * *$ - significativos a $10 ; 5 ; 1$ e $0,1 \%$ de probabilidade, respectivamente, pelo teste $t$

antes da aplicação dos tratamentos, $3,10 \mathrm{mg} \mathrm{dm}^{-3}$, classificados, segundo Alvarez et al. (1999) como muito bom e bom, respectivamente. Barbosa et al. $(2002 ; 2007)$ constataram, a exemplo do observado nesta pesquisa, efeito residual da aplicação de doses crescentes de lodo de esgoto o qual promoveu aumento nos teores de cálcio do solo até a dose de $36 \mathrm{Mg} \mathrm{ha}^{-1}$ de lodo de esgoto.

O composto de lodo de esgoto apresentou efeito residual sobre os teores de magnésio no solo apenas na camada de 0$10 \mathrm{~cm}$ de profundidade (Tabela 4). Nesta camada os teores de $\mathrm{Mg}$ no solo aumentaram com o incremento das doses de lodo de esgoto até atingir a dose de 45,63 $\mathrm{Mg} \mathrm{ha}^{-1}$. Com a dose de máxima produtividade do milho $\left(75 \mathrm{Mg} \mathrm{ha}^{-1}\right)$, o teor deste elemento na camada superficial foi de $1,15 \mathrm{cmol} \mathrm{dm}^{-3}$, classificado como bom, segundo Alvarez et al. (1999), correspondendo à mesma classificação dos teores no solo antes da instalação do experimento. $\mathrm{O}$ aumento dos teores de magnésio no solo em razão do efeito residual de doses crescentes de lodo de esgoto, também foi constatado por Barbosa et al. (2002; 2007).

Observa-se, na Tabela 5, que aumentos nas doses de composto de lodo de esgoto proporcionaram aumentos lineares nos teores de fósforo e nitrogênio nas folhas de milho confirmando que, mesmo após um ano de sua aplicação no solo e duas safras consecutivas de milho, o lodo de esgoto ainda disponibiliza esses nutrientes às plantas. Gomes et al. (2007) constataram aumentos nos teores foliares de nitrogênio em folhas de milho, em razão da adubação com lodo de esgoto; entretanto não observaram efeito deste fertilizante sobre os teores foliares de fósforo. Apesar do aumento dos teores de fósforo e de nitrogênio, a maior dose do composto não foi suficiente para elevar seus teores a níveis de nutrição considerados adequados (Oliveira, 2004); contudo, não foram verificados sintomas visuais de deficiência desses elementos na planta.

O teor de potássio na folha de milho aumentou com o incremento das doses de composto de lodo de esgoto, até a aplicação de 34,51 $\mathrm{Mg} \mathrm{ha}^{-1}$ (Tabela 5). Com a aplicação de 75 $\mathrm{Mg} \mathrm{ha}^{-1}$, dose que gerou máxima produtividade do milho, o teor deste elemento na folha foi de $0,92 \mathrm{mg} \mathrm{dm}^{-3}$ valor que se encontra abaixo da faixa que indica concentrações nutricionais adequadas de potássio na folha de milho (Oliveira, 2004). Tal fato foi observado também por Silva et al. (2002a); entretanto, tal como na presente pesquisa, as plantas não apresentaram sintomas visuais de deficiência.

Os teores de cálcio, de magnésio e de enxofre na folha não foram influenciados pela aplicação do composto de lodo. Embora o teor de Ca não tenha apresentado relação com o aumento da dose de composto de lodo de esgoto, o valor observado nas folhas encontra-se dentro dos limites considerados adequados, conforme Oliveira (2004). O mesmo não foi observado para magnésio e enxofre, que apresentaram valores inferiores aos considerados suficientes para suprir a demanda nutricional da cultura apesar de, a exemplo dos outros nutrientes, não terem sido observados sintomas visuais de 
Tabela 5. Equações de regressão relacionando os teores de nutrientes na fol ha de milho e as doses residuais de composto de lodo de esgoto aplicadas

\begin{tabular}{|c|c|c|c|c|c|c|}
\hline $\begin{array}{l}\text { Nutriente } \\
\left(\text { dag } \mathrm{kg}^{-1}\right)\end{array}$ & Equação & $\mathbf{R}^{2}$ & $\begin{array}{c}\text { DC } \\
\left(\mathrm{Mg} \mathrm{ha}^{-1}\right)\end{array}$ & $\begin{array}{c}\text { TNP } \\
\left(\mathrm{g} \mathrm{kg}^{-1}\right)\end{array}$ & $\begin{array}{c}\text { TMP } \\
\left(\mathrm{g} \mathrm{kg}^{-1}\right)\end{array}$ & FSN \\
\hline $\mathrm{N}$ & $Y=1,33+0,0038 * X$ & 0,7487 & 75,00 & 16,2 & 16,2 & $2,50-3,5$ \\
\hline$P$ & $Y=0,21+0,0012 * * * X$ & 0,9000 & 75,00 & 3,0 & 3,0 & $1,80-3,0$ \\
\hline $\mathrm{K}$ & $Y=\left(0,92+0,010528^{\circ} X-0,0001525^{\circ} X^{2}\right)^{0,5}$ & 0,6307 & 34,51 & 10,5 & 9,2 & $1,30-3,0$ \\
\hline $\mathrm{Ca}$ & $Y=Y m=0,62$ & - & - & 6,2 & 6,2 & $0,25-1,0$ \\
\hline $\mathrm{Mg}$ & $Y=Y m=0,13$ & - & - & 1,3 & 1,3 & $0,15-0,5$ \\
\hline $\mathrm{S}$ & $Y=Y m=0,13$ & - & - & 1,3 & 1,3 & $0,14-0,3$ \\
\hline
\end{tabular}

DC - dose de compos to de lodo de esgoto para atingir a maior concentração de nutriente na planta, dentro do intervalo experimental

TNP - teor máximo de nutriente na planta, dentro do intervalo experimental

FSN - Faixa de suficiência de nutrientes na planta, de acordo com Oliveira (2004)

TMP - teor do nutriente na folha de milho com a aplicação da dose de composto de lodo de esgoto que gerou a máxima produtividade de milho

, *,**,*** - significativos a 10; $5 ; 1$ e $0,1 \%$ de probabilidade, respectivamente, pelo teste $t$

Tabela 6. Interval os de confiança das médias de produtividade e dos teores de nutrientes no solo e na folha, nos dois cultivos sucessivos de milho

\begin{tabular}{|c|c|c|c|c|c|c|c|c|c|}
\hline \multirow{2}{*}{ Variável } & \multicolumn{2}{|c|}{10 plantio } & \multicolumn{2}{|c|}{20 plantio } & \multirow{2}{*}{ Variável } & \multicolumn{2}{|c|}{ 10 plantio } & \multicolumn{2}{|c|}{ 20 plantio } \\
\hline & Média & $\mathrm{IC}^{1}$ & Média & IC $^{1}$ & & Média & $I^{1}$ & Média & $I^{1}$ \\
\hline Produtividade $\left(\mathrm{Mg} \mathrm{ha}^{-1}\right)$ & 4,10 & $\pm 0,64$ & 2,75 & $\pm 0,34$ & $\mathrm{Ca}(0-10 \mathrm{~cm})\left(\mathrm{cmol}_{\mathrm{c}} \mathrm{dm}^{-3}\right)$ & 5,23 & $\pm 0,55$ & 5,72 & $\pm 0,36$ \\
\hline $\mathrm{N}(0-10 \mathrm{~cm})\left(\mathrm{mg} \mathrm{dm}^{-3}\right)$ & 131,35 & $\pm 15,46$ & 147,12 & $\pm 21,49$ & $\mathrm{Ca}(10-20 \mathrm{~cm})\left(\mathrm{cmol}_{\mathrm{c}} \mathrm{dm}^{-3}\right)$ & 3,12 & $\pm 0,38$ & 3,45 & $\pm 0,45$ \\
\hline $\mathrm{N}(10-20 \mathrm{~cm})\left(\mathrm{mg} \mathrm{dm}^{-3}\right)$ & 128,49 & $\pm 18,42$ & 136,44 & $\pm 25,98$ & $\mathrm{Ca}(20-30 \mathrm{~cm})\left(\mathrm{cmol}_{\mathrm{c}} \mathrm{dm}^{-3}\right)$ & 1,43 & $\pm 0,19$ & 1,71 & $\pm 0,27$ \\
\hline $\mathrm{N}(20-30 \mathrm{~cm})\left(\mathrm{mg} \mathrm{dm}^{-3}\right)$ & 115,94 & $\pm 12,00$ & 133,49 & $\pm 18,81$ & $\mathrm{Ca}(30-40 \mathrm{~cm})\left(\mathrm{cmol}_{\mathrm{c}} \mathrm{dm}^{-3}\right)$ & 0,88 & $\pm 0,12$ & 1,11 & $\pm 0,16$ \\
\hline $\mathrm{N}(30-40 \mathrm{~cm})\left(\mathrm{mg} \mathrm{dm}^{-3}\right)$ & 105,46 & $\pm 22,88$ & 130,86 & $\pm 20,91$ & $\mathrm{Ca}(40-50 \mathrm{~cm})\left(\mathrm{cmol}_{\mathrm{c}} \mathrm{dm}^{-3}\right)$ & 0,62 & $\pm 0,10$ & 0,85 & $\pm 0,28$ \\
\hline $\mathrm{N}(40-50 \mathrm{~cm})\left(\mathrm{mg} \mathrm{dm}^{-3}\right)$ & 102,23 & $\pm 11,53$ & 163,53 & $\pm 32,30$ & $\mathrm{Mg}(0-10 \mathrm{~cm})\left(\mathrm{cmol}_{\mathrm{c}} \mathrm{dm}^{-3}\right)$ & 1,00 & $\pm 0,24$ & 1,15 & $\pm 0,06$ \\
\hline$P(0-10 \mathrm{~cm})\left(\mathrm{mg} \mathrm{dm}^{-3}\right)$ & 34,25 & $\pm 19,24$ & 8,66 & $\pm 1,55$ & $\mathrm{Mg}(10-20 \mathrm{~cm})\left(\mathrm{cmol}_{\mathrm{c}} \mathrm{dm}^{-3}\right)$ & 0,81 & $\pm 0,21$ & 0,83 & $\pm 0,09$ \\
\hline$P(10-20 \mathrm{~cm})\left(\mathrm{mg} \mathrm{dm}^{-3}\right)$ & 9,54 & $\pm 5,41$ & 3,68 & $\pm 1,54$ & $\mathrm{Mg}(20-30 \mathrm{~cm})\left(\mathrm{cmol}_{\mathrm{c}} \mathrm{dm}^{-3}\right)$ & 0,00 & $\pm 0,00$ & 0,48 & $\pm 0,06$ \\
\hline$P(20-30 \mathrm{~cm})\left(\mathrm{mg} \mathrm{dm}^{-3}\right)$ & 2,64 & $\pm 1,09$ & 1,15 & $\pm 0,47$ & $\mathrm{Mg}(30-40 \mathrm{~cm})\left(\mathrm{cmol}_{\mathrm{c}} \mathrm{dm}^{-3}\right)$ & 0,00 & $\pm 0,00$ & 0,30 & $\pm 0,06$ \\
\hline$P(30-40 \mathrm{~cm})\left(\mathrm{mg} \mathrm{dm}^{-3}\right)$ & 1,45 & $\pm 0,86$ & 0,76 & $\pm 0,50$ & $\mathrm{Mg}(40-50 \mathrm{~cm})\left(\mathrm{cmol}_{\mathrm{c}} \mathrm{dm}^{-3}\right)$ & 0,00 & $\pm 0,00$ & 0,25 & $\pm 0,07$ \\
\hline$P(40-50 \mathrm{~cm})\left(\mathrm{mg} \mathrm{dm}^{-3}\right)$ & 1,04 & $\pm 0,57$ & 0,58 & $\pm 0,22$ & $\mathrm{~N}$-folha $\left(\right.$ dag $\left.\mathrm{kg}^{-1}\right)$ & 1,33 & $\pm 0,06$ & 1,47 & $\pm 0,12$ \\
\hline $\mathrm{K}(0-10 \mathrm{~cm})\left(\mathrm{mg} \mathrm{dm}^{-3}\right)$ & 148,67 & $\pm 16,22$ & 100,17 & $\pm 11,95$ & P-folha (dag $\mathrm{kg}^{-1}$ ) & 0,15 & $\pm 0,01$ & 0,25 & $\pm 0,04$ \\
\hline $\mathrm{K}(10-20 \mathrm{~cm})\left(\mathrm{mg} \mathrm{dm}^{-3}\right)$ & 79,08 & $\pm 12,64$ & 53,04 & $\pm 9,75$ & K-folha (dag $\mathrm{kg}^{-1}$ ) & 1,46 & $\pm 0,12$ & 0,99 & $\pm 0,08$ \\
\hline $\mathrm{K}(20-30 \mathrm{~cm})\left(\mathrm{mg} \mathrm{dm}^{-3}\right)$ & 39,42 & $\pm 4,97$ & 31,42 & $\pm 6,74$ & Ca-folha (dag kg $\left.{ }^{-1}\right)$ & 0,74 & $\pm 0,09$ & 0,62 & $\pm 0,04$ \\
\hline $\mathrm{K}(30-40 \mathrm{~cm})\left(\mathrm{mg} \mathrm{dm}^{-3}\right)$ & 28,54 & $\pm 7,06$ & 23,21 & $\pm 6,29$ & Mg-folha (dag $\mathrm{kg}^{-1}$ ) & 0,13 & $\pm 0,01$ & 0,13 & $\pm 0,02$ \\
\hline $\mathrm{K}(40-50 \mathrm{~cm})\left(\mathrm{mg} \mathrm{dm}^{-3}\right)$ & 24,42 & $\pm 5,93$ & 22,37 & $\pm 6,56$ & S-folha (dag $\left.\mathrm{kg}^{-1}\right)$ & 0,13 & $\pm 0,02$ & 0,13 & $\pm 0,01$ \\
\hline
\end{tabular}

Intervalo de confiança da média a 0,05 de probabilidade pelo teste $t$

deficiência na planta. Resultados semelhantes foram observados por Silva et al. (2002a) avaliando o efeito residual de lodo de esgoto na produtividade e na nutrição do milho durante três plantios consecutivos.

Na Tabela 6 observa-se que a produtividade do milho no segundo ciclo correspondeu a apenas $67,07 \%$ da produção alcançada no primeiro cultivo. Os fatores determinantes para esta redução parecem ter sido o fósforo e o potássio, os quais tiveram seus teores no solo razoavelmente diminuídos do primeiro para o segundo cultivo. Entretanto se constata no tecido foliar, que apenas o potássio sofreu redução na sua concentração do primeiro para o segundo cultivo, o que pode diferenciá-lo como o fator mais limitante em relação ao segundo cultivo do milho, considerando-se o aproveitamento dos resíduos de adubação do cultivo anterior. Ante o exposto recomendam-se, sempre, novas adubações com lodo de esgoto, a cada cultivo realizado.

Ficou evidente, também, na Tabela 6, que do primeiro para o segundo cultivo houve movimentação de magnésio, o qual só surgiu na faixa de camadas de 20 a $50 \mathrm{~cm}$, no segundo cultivo. Portanto, este elemento requer manejo especial na adubação com lodo de esgoto devido à sua maior mobilidade no solo quanto pelas baixas concentrações normalmente encontradas neste resíduo.

\section{CONCLUSÕES}

1. Em geral, a produtividade e os teores de nutrientes no solo e na planta no segundo cultivo sucessivo de milho não são influenciados pela adubação com fosfato natural reativo, em solo contendo teor inicial médio de cálcio e irrigado com água de origem calcária.

2. A produtividade de milho e os teores de nutrientes no solo e nas folhas de milho em segundo cultivo sucessivo, aumentam, comumente, com a adição de composto de lodo de esgoto ao solo, sendo a dose de $75 \mathrm{Mg} \mathrm{ha}^{-1}$ a de maior efeito residual.

3. A produtividade do milho é menor no segundo cultivo sucessivo em razão do empobrecimento do solo em fósforo e potássio, recomendando-se novas adubações com lodo de esgoto, a cada cultivo realizado.

\section{AgradeCIMENTOS}

Os autores expressam seus maiores agradecimentos à FAPEMIG, ao CNPq e ao PROCAD/CAPES, pelo apoio financeiro e bolsa de produtividade em pesquisa, que possibilitaram a realização deste trabalho. 


\section{LITERATURA CITADA}

Albuquerque, P. E. P.; Costa, E. L.; Rodrigues, M. G. V.; Maeno, P.; Couto, L. Manejo da irrigação em cultura de milho no projeto Jaíba, Minas Gerais. Sete Lagoas: EPAMIG, EMBRAPA, CODEVASF, BNB, 2000. 6p.

Alvarez V., V. H.; Novais, R. F.; Barros, N. F.; Cantarutti, R. B.; Lopes, A. S. Interpretação dos resultados das análises de solos. In: Ribeiro A. C.; Guimarães P. T. G.; Alvarez V., V. H. (org.) Recomendações para o uso de corretivos e fertilizantes em Minas Gerais: 5a aproximação. Viçosa: Comissão de Fertilidade do Solo do Estado de Minas Gerais. 1999. p.2532.

Barbosa, G. M. de C.; Tavares Filho, J.; Brito, O. B.; Fonseca, I. C. B. Efeito residual do lodo de esgoto na produtividade do milho safrinha. Revista Brasileira de Ciência de Solo, v.31, p.601-605, 2007.

Barbosa, G. M. de C.; Tavares Filho, J.; Fonseca, I. C. de B. Propriedades químicas de um Latossolo Vermelho Eutroférrico após aplicação por dois anos consecutivos de lodo de esgoto. Acta Scientiarum, v.24, p.1501-1505, 2002.

Biondi, C. M.; Nascimento, C. W. A. do. Acúmulo de nitrogênio e produção de matéria seca de plantas em solos tratados com lodo de esgoto. Revista Caatinga, v.18, p.123-128, 2005.

Bordin, I.; Neves, C. S. V. J.; Medina, C. de C.; Santos, J. C. F. dos; Torres, E.; Urquiaga, S. Matéria seca, carbono e nitrogênio de raízes de soja e milho em plantio direto e convencional. Pesquisa Agropecuária Brasileira, v.43, p.1785-1792, 2008.

Chiba, M. K.; Mattiazzo, M. E.; Oliveira, F. C. Cultivo de canade-açúcar em Argissolo tratado com lodo de esgoto. I Disponibilidade de nitrogênio no solo e componentes de produção. Revista Brasileira de Ciência de Solo, v.32, p.643652, 2008a.

Chiba, M. K.; Mattiazzo, M. E.; Oliveira, F. C. Cultivo de canade-açúcar em Argissolo tratado com lodo de esgoto: II Fertilidade do solo e nutrição da planta. Revista Brasileira de Ciência de Solo, v.32, p.653-662, 2008b.

Corrêa, R. M.; Nascimento, C. W. A. do; Souza, S. K. de S.; Freire, F. J.; Silva, G. B. da. Gafsa rock phosphate and triple superphosphate for dry matter production and $\mathrm{P}$ uptake by corn. Scientia Agricola, v.62, p.159-164, 2005.

Duete, R. R. C.; Muraoka, T.; Silva, E. C. da; Ambrosano, E. J.; Trivelin, P. C. O. Acúmulo de nitrogênio (15n) pelos grãos de milho em função da fonte nitrogenada em latossolo vermelho. Bragantia, v.68, p.463-472, 2009.

EMBRAPA - Empresa Brasileira de Pesquisa Agropecuária. Manual de métodos de análise de solo. 2.ed. Rio de Janeiro: Embrapa CNPS, 1997. 212p.

Ernani, P. R.; Almeida, J. A.; Santos, F. C. Potássio. In: Novais, R. F.; Alvarez, V. V. H.; Barros, N. F.; Fontes, R. L. F.; Cantarutti, R. B.; Neves, J. C. L. (ed.) Fertilidade do Solo. Viçosa: Sociedade Brasileira de Ciência do Solo, 2007. p.551594.

Gomes, S. B. V.; Nascimento, C. W. A.; Biondi, C. M. Produtividade e composição mineral de plantas de milho em solo adubado com lodo de esgoto. Revista Brasileira de Engenharia Agrícola e Ambiental, v.11, p.459-465, 2007.
Lemainski, J.; Silva, J. E. da. Utilização do biossólido da CAESB na produção de milho no Distrito Federal. Revista Brasileira de Ciência de Solo, v.30, p.741-750, 2006a.

Lemainski, J.; Silva, J. E. da. Avaliação agronômica e econômica da aplicação de biossólido na produção de soja. Pesquisa Agropecuária Brasileira, Brasília, v.41, p.1477-1484, 2006b.

Malavolta, E.; Vitti, G. C.; Oliveira, S. A. Avaliação do estado nutricional das plantas: princípios e aplicações. 2.ed. Piracicaba: POTAFOS, 1997.319p.

Martins, A. L. C.; Bataglia, O. C.; Camargo, O. A.; Cantarella, H. Produção de grãos e absorção de $\mathrm{Cu}, \mathrm{Fe}, \mathrm{Mn}$ e $\mathrm{Zn}$ pelo milho em solo adubado com lodo de esgoto, com e sem calcário. Revista Brasileira de Ciência de Solo, v.27, p.563574, 2003.

Nascimento, C. W. A.; Barros, D. A. S.; Melo, E. E. C.; Oliveira, A. B. Alterações químicas em solos e crescimento de milho e feijoeiro após aplicação de lodo de esgoto. Revista Brasileira de Ciência de Solo, v.28, p.385-392, 2004.

Noce, M. A. Milho variedade BR 106: Técnicas de plantio. Sete Lagoas: Embrapa Milho e Sorgo, 2004. 5p. Comunicado Técnico, 109.

Nogueira, T. A. R.; Sampaio, R. A., Ferreira, C. S.; Fonseca, I. M. Produtividade de milho e de feijão consorciados adubados com diferentes formas de lodo de esgoto. Revista de Biologia e Ciências da Terra, v.6, p.122-131, 2006.

Novais, R. F.; Almeida, J. A.; Santos, F. C. Fósforo. In: Novais, R. F.; Alvarez V., V. H.; Barros, N. F.; Fontes, R. L. F.; Cantarutti, R. B.; Neves, J. C. L. (ed.) Fertilidade do Solo. Viçosa: Sociedade Brasileira de Ciência do Solo, 2007. p.471550.

Novais, R. F.; Smith, T. J. Fósforo em solo e planta em condições tropicais. 1.ed. Viçosa: UFV, DPS, 1999. 399p.

Oliveira, S. A. Análise foliar. In: Sousa, D. M. G.; Lobato, E. Cerrado: correção do solo e adubação. Planaltina: Embrapa Cerrados. 2004. p.245-256.

Resende, A. V.; Furtini Neto, A. E.; Alves, V. M. C.; Muniz, J. A.; Curi, N.; Lago, F. J.; Resposta do milho a fontes e modos de aplicação de fósforo durante três cultivos sucessivos em solo da região do cerrado. Ciência Agrotécnica, v.30, p.458466, 2006.

Silva. F. C. da; Boaretto, A. E.; Berton, R. S.; Zotelli, H. B.; Pexe, C. A.; Bernardes. E. M. Efeito de lodo de esgoto na fertilidade de um Argissolo Vermelho-Amarelo cultivado com canade-açúcar. Pesquisa Agropecuária Brasileira, v.36, p.831-840, 2001.

Silva, J. E.; Resck, D. V. S.; Sharma, R. D. Alternativa agronômica para o biossólido produzido no Distrito Federal. I. Efeito na produção de milho e na adição de metais pesados em Latossolo no Cerrado. Revista Brasileira de Ciência de Solo, v.26, p.487-495, 2002a.

Silva, J. E.; Resck, D. V. S.; Sharma, R. D. Alternativa agronômica para o biossólido produzido no Distrito Federal. II. Aspectos qualitativos, econômicos e práticos de seu uso. Revista Brasileira de Ciência de Solo, v.26, p.497-503, 2002 b.

Tedesco, M. J.; Gianello, C.; Bissani, C. A.; Bohnen, H.; Volkweiss, S. J. Análise de solo, plantas e outros materiais. 2.ed. Porto Alegre: Departamento de Solos/UFRGS. 1995. 174p. Boletim Técnico, 5 
Trannin, I. C. de B.; Siqueira, J. O.; Moreira, F. M. de S. Avaliação agronômica de um biossólido industrial para a cultura do milho. Pesquisa Agropecuária Brasileira, v.40, p.261-269, 2005.
Vieira, R. F.; Tanaka, R. T.; Tsai, S. M.; Pérez, D. V.; Silva, C. M. M. de S. Disponibilidade de nutrientes no solo, qualidade de grãos e produtividade da soja em solo adubado com lodo de esgoto. Pesquisa Agropecuária Brasileira, v.40, p.919-926, 2005. 